\title{
Invasive Techniques in Scar Management: Skin Substitutes
}

F. W. Timmermans and E. Middelkoop

\section{Contents}

37.1 Background - 318

37.2 Permanent Wound Coverage - $\mathbf{3 1 8}$

37.2.1 Epidermal Cells/Cultured Epidermal Autografts CEA - 318

37.2.2 Dermal Substitutes - 320

37.2.3 Cellular Dermal Substitutes - 321

37.3 Full-Skin Substitutes -321

37.4 Subcutaneous Fat - 321

37.5 Regulatory/Safety Issues - 322

37.6 Conclusion - 322

References -323 


\subsection{Background}

Modern wound care demands a high focus on quality of healing. Skin substitutes can play an important role in achieving the best possible outcome for each patient. Although skin substitutes were primarily developed for use in acute wound care, they have since then started to play an important role in secondary scar revision surgery. Initially, epidermal cell cultures were developed with a focus on efficient wound closure of large wound surfaces. Long-term results, however, indicated that several structures that played a role in stabilizing the connection between epidermis and dermis did not fully regenerate [1]. Thus the role and importance of the dermal component of skin regeneration became more apparent. This eventually led to the development of dermal substitutes. Various biomaterials were tested for their capacity to stimulate dermal regeneration, with various degrees of success. Synthetic as well as biological materials were extensively tested. Where synthetic materials demonstrated to actively stimulate wound healing in chronic circumstances such as diabetic wounds, biological biomaterials proved to be more beneficial for treating acute wounds. These biological materials were mainly composed of purified extracellular skin matrix components such as collagen, elastin, and/ or proteoglycans. Long-term clinical results of several of these materials have since then become available. Further development of the field resulted in the addition of cells to these synthetic and biomaterial constructs. Various cell sources were investigated, with specific attention to different sources of stem cells, as well as allogeneic versus autologous cell sources [2-4]. Alongside the clinical studies, outcome analysis on different skin and scar parameters was further developed. This provided insight into the different skin characteristics that were influenced by the use of skin substitutes. Besides acute wound treatment, scar treatment using fat-derived mesenchymal stem cells is also subject of further research. Initial studies have demonstrated beneficial effects on scar maturation after lipofilling. This technique aims at regeneration of the subcutaneous fat layer underneath the dermal layer of the skin. Nowadays, all these developments allow us to use these different biomaterials as acellular dermal substitutes in state-ofthe-art reconstructive surgery and acute wound treatment. New advancements are found in the development of full skin constructs, using autologous epidermal as well as dermal cell sources. At present, autologous (i.e., from the patient) cells are still the mainstay in the successful use of cellular substitutes. This is an advantage in terms of the absence of potential immunological reactions, but at the same time, the long culture times needed to prepare these constructs are definitely a disadvantage of using cellular substitutes in the acute trauma setting. Furthermore, the long-term regenerative capacity of these cellular skin constructs still remains to be fully established.

\subsection{Permanent Wound Coverage}

Wound closure normally proceeds by migrating epidermal cells from both wound edges and skin appendages such as sebaceous glands and hair follicles. As wounds grow deeper and larger, the skin loses its ability to provide enough epidermal cells to close the wounds in a reasonable time frame. This time frame of healing is considered to be adequate if the wound is closed within 3-4 weeks. Prolonged exposure to pathogens and fluid loss represent immediate dangers in delayed healing and might potentially lead to bacterial contamination, sepsis, and death in the worst cases. Biological processes such as wound contraction are initiated that aimed at speeding up wound closure. This is paradoxically an undesired action in terms of the final quality of the wound-healing process. Therefore, most large and deep wounds require surgical assistance to promote timely wound healing and adequate wound closure. The main purpose of surgery is to bring a new source of viable epidermal cells to the wound in order to speed up the wound closure. Skin grafts are the most well-known and most used methods for epidermal transfer and permanent wound coverage.

\subsubsection{Epidermal Cells/Cultured Epidermal Autografts CEA}

The keratinocytes in the epidermal layer of the skin form the first barrier of the skin. A standard technique for closure of large wounds is transplantation of skin using a split-thickness skin graft (STSG). The protective properties of the epidermal layer are best conserved in the form of split-thickness skin grafts, which usually comprise the whole of the epidermis and a very thin layer of dermis (approximately $0.1-0.2 \mathrm{~mm}$ ). Coverage of larger wound areas is possible using meshed STSGs, where expansion rates up to 1:6 are possible (• Fig. 37.1). However, scar formation in the mesh interstices is apparent, and therefore larger expansions with fewer donor sites were needed. This pushed Rheinwald and Green, as early as 1975, into being the first to show that keratinocytes could be extracted, in vitro cultured into sheets, and that they were suitable for grafting [5]. This can easily be considered the first step in the tissue engineering of the skin. The usage of cultured cells marked a leap forward in the treatment 

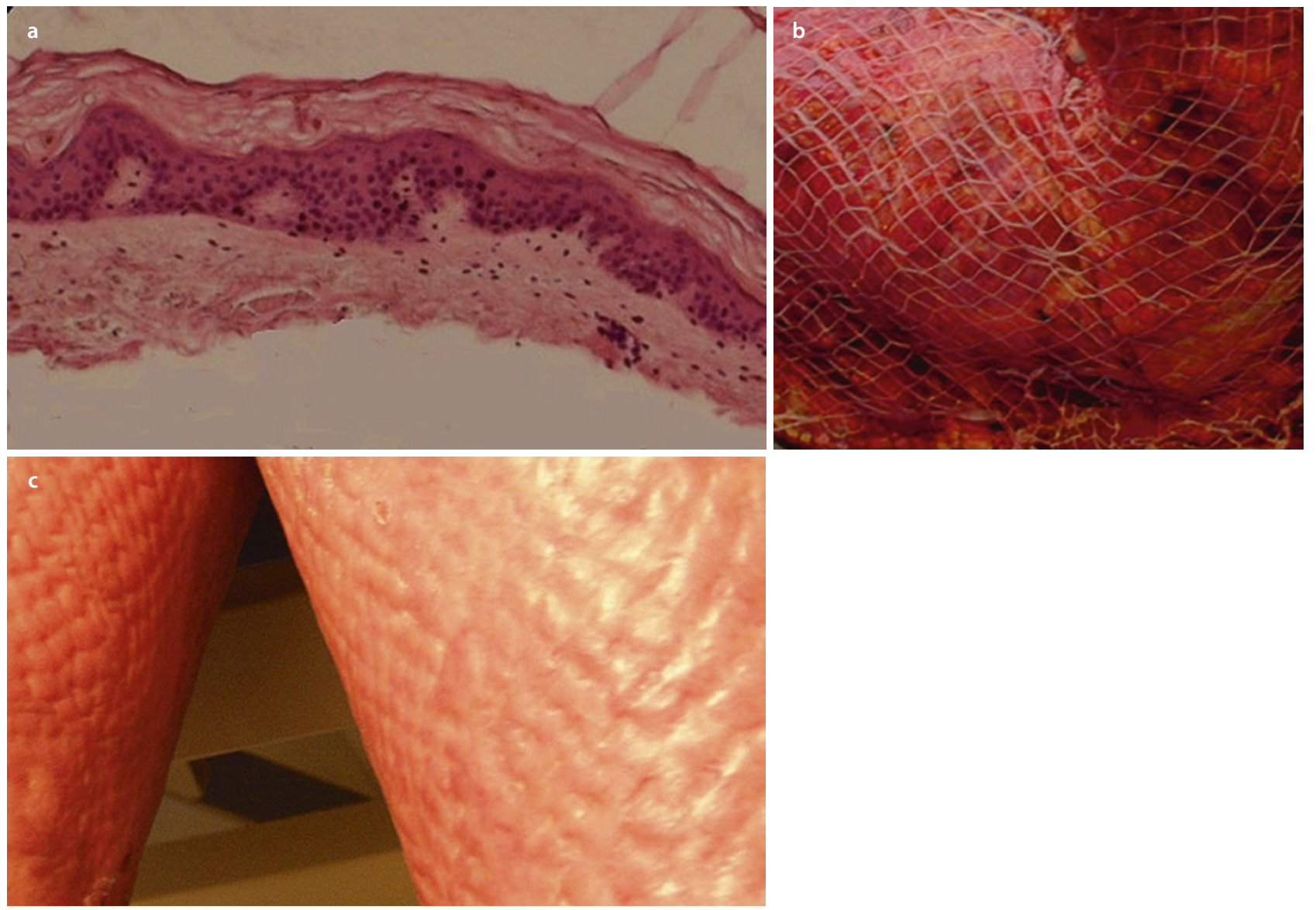

- Fig. 37.1 a Microscopic image of hematoxylin eosin staining of human split skin. Note that only a very thin layer of dermis is present. b Split-skin mesh graft with wide expansion to cover larger wound area. $\mathbf{c}$ Typical scar pattern showing mesh pattern of the graft in the scar

of burn patients as larger areas of wounds could be covered with very small donor sites of healthy skin.

The development of cell culture techniques opened up the possibility to maintain viable cells outside of the human body. Initially, cultured keratinocyte layers (cultured epithelial autografts or CEA) opened opportunities to cover large parts of the body without the necessity for substantial donor sites. This greatly improved the survival chances of severely burned patients. However, early studies of CEAs on wound beds showed insufficient regeneration of dermal-epidermal junctions between the epidermis and the underlying tissue, which even after many years resulted in a vulnerable and weak skin barrier [1]. Thus, it was discovered that substitution of dermal tissue is also of great importance in creating and maintaining a well-functioning new skin. These insights sparked the development of more complex skin substitutes.

Although CEAs have been used for over 40 years now, they did not provide the definitive solution for the treatment of large wounds. Specific downsides such as variable graft-take rates, high manufacturing costs, production and transportation logistics, insufficient der- mal-epidermal junction regeneration, and often having to compete with readily available split-skin grafts have limited most of the further development of CEAs [6].

A more recent development in the application of epidermal cells is in the form of in situ cell therapy, called the $\mathrm{ReCell}^{\circledR}$ system (Avita Medical, Cambridge, UK). With only a small donor site of split skin (approximately $1 \mathrm{~cm}^{2}$ ), this device allows the isolation of a single-cell suspension that can be sprayed on a wound bed. The cell suspension consists of approximately $65 \%$ keratinocytes, $30 \%$ fibroblasts, and 3-5\% melanocytes. This system avoids the time-consuming and costly cell culture step. The cells in the cell suspension can adhere to the wound bed and promote tissue regeneration by both cell proliferation and growth factor stimulation. This technique is sometimes used in combination with expanded meshed autografts to accelerate wound healing. The combination of these two treatment options has shown to improve cosmetic outcomes of scars and pigment abnormalities. In situ cell therapy can also be used as a means to promote repigmentation of depigmented skin in patients with vitiligo and congenital melanocytic naevi [7]. Whether these effects are due to actual cell 
survival and proliferation or to growth factor and cytokine secretion is presently still unclear.

\subsubsection{Dermal Substitutes}

Skin substitutes aiming at dermal regeneration are dermal constructs designed to either temporarily or permanently replace dermal defects. In all cases, the aim of skin substitutes is to provide protection against microorganisms, reduce pain, promote wound healing, and assist in recreating the barrier function of the skin [8]. This method has become increasingly important for the treatment of burns, open wounds, chronic wounds, and deep tissue donor sites, as it has been shown to improve and accelerate skin regeneration, reduce scar contracture, and minimize donor site morbidity. All these different treatment indications resulted in the development of a wide range of skin substitutes with different characteristics such as mono- or bilayered compositions, temporary or permanent fixture, and cellular or acellular skin substitutes.

\subsubsection{Tissues}

A biological approach used in the design of permanent skin substitutes was to start with real human tissue. One of the early developed acellular allogeneic skin substitutes is Alloderm ${ }^{\circledR}$. Alloderm ${ }^{\circledR}$ is harvested from cadaver donors as skin grafts, which are then washed with hypertonic saline to remove cell remnants. After removal of the epithelial layer, the remaining dermal layer is treated to inactivate viruses and then freeze-dried to be used on demand. This provides a nonantigenic dermal scaffold with basement membrane proteins, which are known to promote wound healing and angiogenesis. After rehydration of the Alloderm ${ }^{\circledR}$, coverage with an ultrathin split skin would suffice as a full coverage treatment option. Recently, Alloderm ${ }^{\circledR}$ has seen a rise in its use for breast reconstructive surgery, whereas the use of Alloderm ${ }^{\circledR}$ on wounds and burns remains limited. Glyaderm ${ }^{\circledR}$ is a similar product based on allogeneic dermis preserved in glycerol instead of freeze-drying. Initial studies on burn patients have been performed with favorable results [9].

\subsubsection{Dermal Scaffolds}

Some of the most well-known and most used dermal scaffolds are Integra ${ }^{\circledR}$ and Matriderm ${ }^{\circledR}$. Although both materials consist of bovine collagen combined with extracellular matrix components, their mode of application is generally different. Integra ${ }^{\circledR}$ was the first dermal substitute of its kind in the early 1980s and was created with the apparent goal of minimizing fluid loss and bacterial contamination and promoting cell migration into the wound bed. It does so through its two-layered com- position and its two-stage procedure. The deeper layer of Integra ${ }^{\circledR}$ is made out of a combination of bovine collagen and glycosaminoglycan chondroitin-6-sulfate, whereas the top is made with a 0.2 -mm-thick polysiloxamine polymer membrane with vapor-transmitting characteristics similar to normal epithelium. This membrane is intended to be placed on the full-thickness wound, and the outer silicone membrane will function as a temporary epidermal replacement and will need to be replaced by a split-skin graft after 2-3 weeks of sufficient ingrowth of vasculature into the deeper dermal scaffold. Due to the combination with a silicone layer as temporary epidermal coverage, Integra ${ }^{\circledR}$ can immediately function as a barrier to limit fluid loss and protect from microbial contamination of the wound, while providing the required extracellular scaffolding for cell ingrowth and proliferation of fibroblasts and endothelial cells. In the second stage, after vascular ingrowth, the silicone layer is replaced by a thin split-skin graft. This product has gained widespread use in the clinical treatment of deep partial-thickness and full-thickness burn wounds, full-thickness skin defects of other etiologies, chronic wounds, and soft tissue defects. The results of Integra ${ }^{\circledR}$ within burn care have been generally favorable and have shown improved quality of healing. However, the staged procedure leaves the wound open for a prolonged period of time and thus bears a risk of submembranous bacterial infections resulting in loss of the biomaterial. Other similar bilayered substitutes are Renoskin ${ }^{\circledR}$, Pelnac ${ }^{\mathrm{TM}}$, and Hyalomatrix ${ }^{\circledR}[10]$.

Matriderm ${ }^{\circledR}$ is usually applied in a one-step procedure as this dermal scaffold allows immediate coverage with a thin split-skin graft. It consists of an extracellular matrix scaffold made out of purified, freeze-dried bovine collagen mixed with 3\% elastin hydrolysate. An autologous split-skin graft can be applied directly onto the Matriderm ${ }^{\circledR}$. Clinical research showed a somewhat slower take of the graft, which reflects the interpositioning of the unvascularized scaffold between the wound bed and the split-skin graft. Nevertheless, the outcome in terms of scar quality was shown to be superior over splitskin graft treatment even after a 12-year follow-up [11].

Both Matriderm ${ }^{\circledR}$ and Integra ${ }^{\circledR}$ have been used for acute and reconstructive purposes and as a means to improve scar quality. These dermal scaffolds initially lack cellular compounds and do require the body to incorporate the exogenous matrix into the wound environment through cell migration and remodeling, which is mediated by macrophages and fibroblasts. Fibroblasts, similar to keratinocytes, also secrete various cytokines and growth factors. But besides promoting cell proliferation, inducing angiogenesis, and modulating the inflammatory process, they also produce a new extracellular matrix, which helps to reconstruct the three-dimensional dermal structure of 
the skin. Other one-layered scaffolds are Novomaix ${ }^{\circledR}$ (freeze-dried collagen with elastin fibers) and Permacol ${ }^{\circledR}$ (a porcine collagen matrix which is now primarily used in hernia and abdominal wall surgery) [10].

\subsubsection{Cellular Dermal Substitutes}

Cellular dermal and composite skin substitutes are the most recent products of skin substitute research. Dermal skin substitutes are primarily created to guide dermal regeneration and are used in combination with autologous split-skin transplants. This treatment is aimed at providing an underlying layer of dermal tissue where normally fibrotic scar tissue would be formed. One of the disadvantages of an acellular dermal scaffold is the relatively slow ingrowth of blood vessels and other cells into the scaffold, which can hamper the take and even minimize the survival of the covering split-skin autograft. The general understanding is that the inclusion of dermal cells in the scaffolds improves these outcomes and at the same time promotes the wound-healing process. DenovoDerm ${ }^{\mathrm{TM}}$, Hyalograft $3 \mathrm{D}^{\circledR}$, Dermagraft ${ }^{\circledR}$, and TransCyte ${ }^{\circledR}$ are a few of these known cellular dermal skin substitutes. The first two are, respectively, made of autologous fibroblast seeded in bovine collagen and hyaluronic acid scaffolds, and the latter two are respectively made of allogeneic fibroblasts seeded in a polyglactin and nylon scaffold. Other experimental studies have looked into using adipose-derived regenerative cells in combination with Integra $^{\circledR}$, and some attempts have been made to create skin substitutes with nanotechnology. However, the effectiveness and safety of these methods still need to be demonstrated outside of the experimental stages of development.

Most of the cellular dermal substitutes are produced using living neonatal fibroblasts. Examples include TransCyte $^{\circledR}$ and Dermagraft ${ }^{\circledR}$. TransCyte is produced by culturing neonatal fibroblasts on a nylon mesh, where the cells deposit collagen, other matrix proteins, and growth factors. After freezing, the cells are no longer alive, but the matrix proteins and growth factors remain and can actively promote healing, e.g., for deep partial thickness acute wounds. Dermagraft ${ }^{\mathbb{R}}$, however, is composed of living allogeneic neonatal fibroblasts cultured on a polyglactin scaffold. The neonatal cells provide the extracellular matrix compounds and growth factors needed for efficient tissue regeneration. The allogeneic compounds in the skin substitute will subsequently stimulate granulation tissue formation that enables secondary closure or eventual coverage with a split-skin graft. Compared to the autologous counterparts, allogenic skin substitutes have thus far predominantly been successfully used for the treatment of chronic diabetic ulcers.

\subsection{Full-Skin Substitutes}

Several research groups have developed full biological skin substitutes comprising of both dermis and epidermis, while containing either allogeneic or autologous fibroblasts and keratinocytes. Apligraf ${ }^{\circledR}$ was the first of this kind to become commercially available. Apligraf ${ }^{\circledR}$ consists of cultured allogenic human foreskin-derived neonatal fibroblasts in a bovine type I collagen matrix over which allogenic human foreskin-derived neonatal epidermal keratinocytes are then cultured and allowed to stratify. The allogeneic character of this skin substitute ultimately results in the rejection of the keratinocytes, which eventually requires an autologous split-skin coverage for definite would closure. Therefore, Apligraf ${ }^{\circledR}$ is less suitable for large acute wounds but has found its main use in the treatment of chronic wounds. Orcel ${ }^{\circledR}$ is a similar product with a similar composition. The allogenic cells are considered to survive up to $2-3$ weeks in the wound bed, and the overall effectiveness of these composite skin substitutes is thought to be based on the excretion of a mix of growth factors and cytokines.

Some novel autologous composite skin substitutes are DenovoSkin ${ }^{\mathrm{TM}}$ and Permaderm ${ }^{\circledR}$. Both of these composite skin substitutes are made with a bovine collagen scaffold. Another described full-skin substitute is the TissueTech Autograft System ${ }^{\mathrm{TM}}$, which is essentially the combination of a dermal skin substitute (Hyalograft $3 D^{\circledR}$ ) with a CEA (Laserskin ${ }^{\circledR}$ ). A major advantage of autologous composite skin substitutes is its one-stage character. Except for the initial skin biopsy from which the autologous cells are harvested and cultured, no secondary procedures such as split-skin transplantations are required. Most of these are newly developed skin constructs, which are still undergoing trials, and none are commercially available at present. Besides regulatory obstacles (see below), other downsides to the further development of autologous composite skin substitutes are the high production costs, long preparation time, and the necessity for a well-organized production-toclinic transfer. Nevertheless, these are important developments, since in the end, these advancements may lead to clinically applicable regenerative full-skin substitutes, comprising of not only dermal and epidermal structures but also vasculature, skin appendages, and even nerves.

\subsection{Subcutaneous Fat}

In recent years, there has been an apparent shift from merely thinking about reconstructing the dermis and epidermis, to the inclusion of the subcutis. It has become more clear that the subcutaneous fat layer plays an essential role in the gliding and mobile functions of the 
skin. This characteristic is especially important in anatomical locations where the skin is relatively thin, such as the back of the hand or the tibia. Scars in these areas can easily fuse to underlying tendons or bones if subcutaneous fat is missing. Recent studies have noted beneficial effects on scar quality of lipo-injection with the patient's own fat, harvested by liposuction. Lipofilling of these scars showed an increase of elastic fibers at the dermopapillary layer, which could account for the improvement of scar quality [4, 12-14]. The sustainability of a mere single treatment of autologous fat grafting in mature adherent scars showed to promote a lasting improvement in scar elasticity and tactile pliability [15]. Although there is not enough solid clinical evidence yet, the data gathered so far indicate that fat grafting could become a new treatment modality in improving scar quality and outcome after burns. Further research should aim at elucidating the best indications, timing, and techniques as well as frequencies of application.

\subsection{Regulatory/Safety Issues}

Further development of cell therapy and tissue engineering for better clinical outcomes with regard to wound healing and the reduction of scar formation is a common recommendation in studies on the use and development of skin substitutes. However, new regulations have become effective during the last decade, which designate cell-based therapy as advanced therapy medicinal products (ATMPs). Practically, this means that cellbased therapies in Europe are under direct assessment of the European Medicines Agency (EMA, a monitoring institute of the European Union), which is dedicated to the scientific evaluation and supervision of market access of medicinal products. In the USA, the Food and Drug Administration (FDA) is the relevant authority, where the Office of Cellular, Tissue, and Gene Therapies (OCTGT) exerts the tasks to evaluate and supervise market access of ATMP products.

The European Regulation (EC) No. 1394/2007 provides the overall framework on production and use of ATMPs within the European Union. According to this regulation, an ATMP is a "medicine for human use that is based on genes, cells or tissue engineering." When elaborating on the definition of tissue-engineered products, such products may contain or consist of engineered cells or tissues, with the purpose to regenerate, repair, or replace a human tissue, and it may contain viable or nonviable cells or tissues of human or animal origin. This means that most of the skin substitutes with living cells (both autologous and allogeneic), adipose tissue, and matrices containing human and/or xenogeneic material fall under the regulatory issues associated with this regulation. Scaffolds made of isolated and/or purified animal or human-derived proteins, however, are classified as medical devices. One of the main features of these regulations is that the production of ATMPs for human use must take place under Good Manufacturing Practice (GMP) conditions. This is translated into high standards of production facilities, which is only possible in a select few centers and represent a considerable administrative burden for importing and exporting ATMPs across international borders. These strict regulatory and production requirements imply intensive collaboration between centers for the further development of ATMP' and for successful commercial exploitation of these novel treatment options.

\subsection{Conclusion}

Acellular skin substitutes have gained a solid position in the treatment options for acute and chronic wounds as well as scar revisions, with improved outcomes in skin quality over standard split-skin graft treatment. Autologous cellular skin substitutes still hold a promise for the future. Tissue repair and regeneration through the use of autologous cells promote superior healing over the known methods. There are still some drawbacks in the production and application of tissue-engineered skin constructs, but further research, automated production process, and harmonizing our treatment practices will bring us closer toward a scarless future.

\footnotetext{
- Take Home Messages

- Acellular dermal substitutes have proven their efficacy in reaching better scar quality compared to split-skin grafting for acute and reconstructive treatment.

- Acellular dermal substitutes can be used in a onestep procedure, providing immediate wound coverage, or two-step approach, which allows prevascularization of the dermal component prior to epithelial grafting.

- Epidermal cell sprays can be used to treat pigment disorders in scars and/or to increase epithelialization rate during acute wound healing

- Fat grafting is used successfully on adherent scars as autologous fat injection to improve scar elasticity and regenerate a gliding layer underneath a scar.

- Cellular dermal and full-skin substitutes are in clinical trials for treatment of acute and reconstructive wounds.
} 


\section{References}

1. Desai MH, et al. Lack of long-term durability of cultured keratinocyte burn-wound coverage: a case report. J Burn Care Rehabil. 1991;12:540-5.

2. Boyce ST, Lalley AL. Tissue engineering of skin and regenerative medicine for wound care. Burns Trauma. 2018;6:4. https:// doi.org/10.1186/s41038-017-0103-y.

3. Akershoek JJ, et al. Cell therapy for full-thickness wounds: are fetal dermal cells a potential source? Cell Tissue Res. 2016;364:83-94. https://doi.org/10.1007/s00441-015-2293-6.

4. Leonardi D, et al. Mesenchymal stem cells combined with an artificial dermal substitute improve repair in full-thickness skin wounds. Burns. 2012;38:1143-50. https://doi.org/10.1016/j. burns.2012.07.028.

5. Rheinwald JG, Green H. Serial cultivation of strains of human epidermal keratinocytes: the formation of keratinizing colonies from single cells. Cell. 1975;6:331-43.

6. Brockmann I, et al. Skin-derived stem cells for wound treatment using cultured epidermal autografts: clinical applications and challenges. Stem Cells Int. 2018;2018:4623615. https://doi. org/10.1155/2018/4623615.

7. Lommerts JE, et al. Autologous cell suspension grafting in segmental vitiligo and piebaldism: a randomized controlled trial comparing full surface and fractional $\mathrm{CO} 2$ laser recipient-site preparations. Br J Dermatol. 2017;177:1293-8. https://doi. org/10.1111/bjd.15569.

8. van der Veen VC, van der Wal MB, van Leeuwen MC, Ulrich MM, Middelkoop E. Biological background of dermal substi- tutes. Burns. 2010;36:305-21. https://doi.org/10.1016/j. burns.2009.07.012.

9. Pirayesh A, Hoeksema H, Richters C, Verbelen J, Monstrey S. Glyaderm $((\mathrm{R}))$ dermal substitute: clinical application and long-term results in 55 patients. Burns. 2015;41:132-44. https:// doi.org/10.1016/j.burns.2014.05.013.

10. van Zuijlen $\mathrm{P}$, et al. Tissue engineering in burn scar reconstruction. Burns Trauma. 2015;3:18. https://doi.org/10.1186/s41038015-0017-5

11. Bloemen MC, van Leeuwen MC, van Vucht NE, van Zuijlen PP, Middelkoop E. Dermal substitution in acute burns and reconstructive surgery: a 12-year follow-up. Plast Reconstr Surg. 2010;125:1450-9. https://doi.org/10.1097/PRS.0b013e3181d62b08.

12. Byrne M, O'Donnell M, Fitzgerald L, Shelley OP. Early experience with fat grafting as an adjunct for secondary burn reconstruction in the hand: technique, hand function assessment and aesthetic outcomes. Burns. 2016;42:356-65. https://doi. org/10.1016/j.burns.2015.06.017.

13. Pallua N, Baroncini A, Alharbi Z, Stromps JP. Improvement of facial scar appearance and microcirculation by autologous lipofilling. J Plast Reconstr Aesthet Surg. 2014;67:1033-7. https:// doi.org/10.1016/j.bjps.2014.04.030.

14. Bruno A, et al. Burn scar lipofilling: immunohistochemical and clinical outcomes. J Craniofac Surg. 2013;24:1806-14. https:// doi.org/10.1097/SCS.0b013e3182a148b9.

15. Jaspers MEH, Brouwer KM, van Trier AJM, Middelkoop E, van Zuijlen PPM. Sustainable effectiveness of single-treatment autologous fat grafting in adherent scars. Wound Repair Regen. 2017;25:316-9. https://doi.org/10.1111/wrr.12521.

Open Access This chapter is licensed under the terms of the Creative Commons Attribution 4.0 International License (http://creativecommons. $\mathrm{org} /$ licenses/by/4.0/), which permits use, sharing, adaptation, distribution and reproduction in any medium or format, as long as you give appropriate credit to the original author(s) and the source, provide a link to the Creative Commons license and indicate if changes were made.

The images or other third party material in this chapter are included in the chapter's Creative Commons license, unless indicated otherwise in a credit line to the material. If material is not included in the chapter's Creative Commons license and your intended use is not permitted by statutory regulation or exceeds the permitted use, you will need to obtain permission directly from the copyright holder.

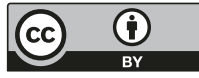

